\title{
Synthesis of Multilayer and Waveguide Filters for use in Optical Communication Systems
}

\author{
Safwat W. Z. Mahmoud, Moustafa F. Ahmed and M. A. Kaid
}

\author{
Department of Physics, Faculty of Science, Minia University, 61519 \\ El-Minia, Egypt.
}

\begin{abstract}
We report on a new synthesis method of reflection filters with multilayer and waveguide structures. The filter is characterized by bandwidth as small as $\leq 1 \mathrm{~nm}$ for effective use in wavelength-division-multiplexed optical communications. Both the transfer-matrix method and the Fourier transformation are employed to determine the filter reflection from a spatially varying refractive index. In contrary to Bragg reflectors that utilize periodic index variations, the present synthesis model employs aperiodic variation around an averaged index. The calculations show that the reflectivity spectrum for 25 pairs of periodic GaAs-AlGaAs quarter-wavelength Bragg stacks is a single band with broad plateau $(\geq 100 \mathrm{~nm})$ surrounded by high lobes $(>80 \%)$. We show that these side-lobes are suppressed to less than 40\% when the same number of layers is used but with an aperiodic varying thickness. Moreover, by exceeding the filter length to $2 \mathrm{~mm}$, we achieved reflectors with $99 \%$ reflectivity, bandwidth of $\leq 1 \mathrm{~nm}$ and low side-lobes $(<-13 \mathrm{~dB})$. Similar characteristics are attained using a GaAs/AlGaAs waveguide with a corrugated structure on the upper interface of the core. The corrugated profile of the core-thickness is obtained for an asymmetric waveguide. We also demonstrate accomplishment of two-band reflectors for use as minus (transmission) filters.
\end{abstract}

\section{Introduction:}

Wavelength-division-multiplexing (WDM) is an efficient technique to enhance the information capacity in optical communications systems [1]. The main function of WDM is the effective use of the fiber bandwidth, which exceeds several terahertz in the low-loss window at $\lambda \sim 1.5 \mu \mathrm{m}$, by simultaneous sending of multiple channels with slightly different wavelengths, as shown schematically in Fig. (1). Optical (transmission or reflection) filters then play important role as wavelength-selective device to route each transmitted signal to the detectors. The bandwidth of the filter should be less than the wavelength separation of fiber channels, which is in the range of $1 \mathrm{~nm}$. Synthesis of high 
output filters with such narrow bandwidth around $1.5 \mu \mathrm{m}$ is a challenge of research.

Design procedures of reflection filters are based on causing spatial variation of the refractive index in such a way to increase the filter reflectively around a desired wavelength by multireflections. The index variation is then translated into variation of an appropriate structure parameter of the filter. Such index variation may be done periodically [2-8], such as the common Bragg reflectors, or aperiodically [9-12], which can then be employed by the transfermatrix method to calculate the reflectivity spectrum [13-16].

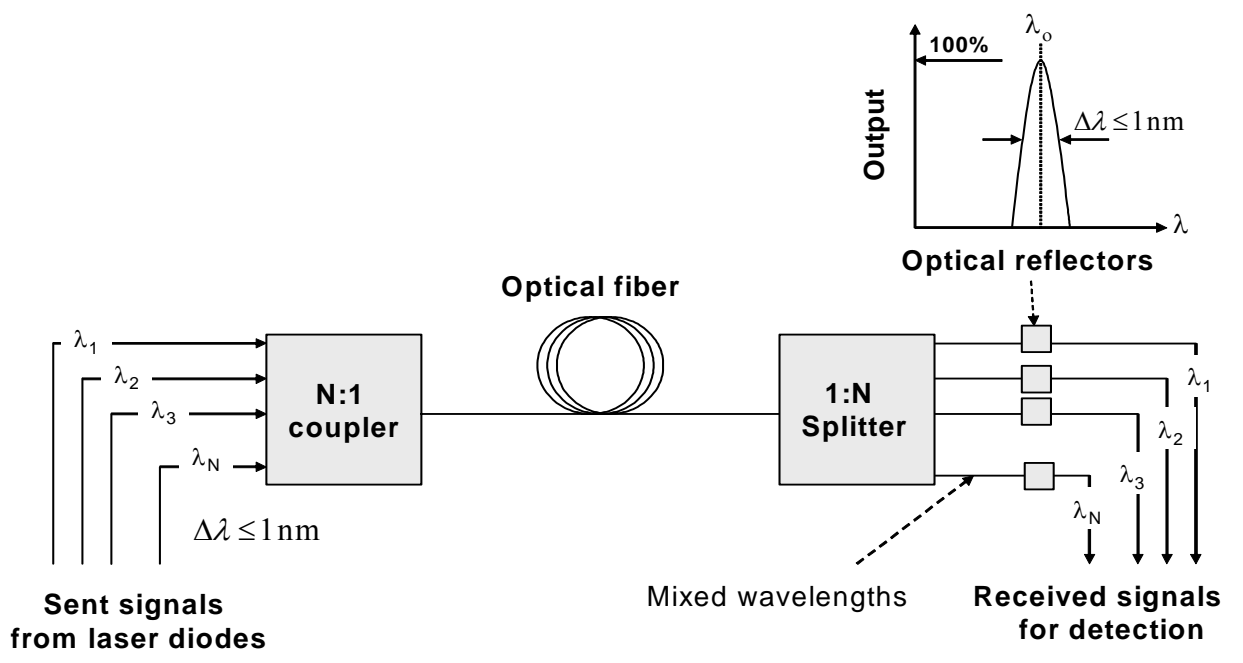

Fig. (1): A scheme of a wavelength-division-multiplexed optical communication system.

On the other hand, the aperiodic-type design helps to reduce the sidelobes, vary the bandwidth and attain output spectrum with multi-bands [14-16]. However, a guide to the profile of the spatial index variation that corresponds to the desired output spectrum is essential to approach to the optimum index profile. Some authors determined this guide as inverse Fourier transformation of the reflection coefficient [14,15], however accuracy of this relationship deteriorates dramatically with decreasing the bandwidth. Then careful techniques are required to optimize such approximate guide of index variation so as to fit the reflectivity spectrum calculated by an accurate method (such as the transfer-matrix method) to desired spectrum [14].

In this paper, we are aimed to introduce a proposal of optical filters with bandwidth as narrow as $\leq 1 \mathrm{~nm}$. The filter configuration is introduced in both 
dielectric multilayer and upper-interface-corrugated waveguide structures normal to the direction of propagation of light. In the former, the index variation is translated into variation in the layer material or thickness, and into variation in the core thickness in the latter. We introduce detailed comparison of employing the periodic Bragg reflectors in the filter design with the present aperiodic structure. In the waveguide filter, we newly introduce the analysis in the general case of asymmetric-waveguide type assuming oscillation of the fundamental transverse mode. We, furthermore, demonstrate accomplishment of two-band reflectors that can be used as minus (transmission) filters.

The paper is structured as follows. In the following section, we introduce the theoretical proposal of the filter design in both multilayer and corrugated waveguide structures. In section 3 , we present the desired spectral characteristics of optical filters. An investigation of periodic Bragg multilayered reflectors and comparison to filters designed with aperiodically-varying refractive index will be given in section 4 . In Addition, we demonstrate the results of the designed filters with bandwidth $\leq 1 \mathrm{~nm}$, which include spectral characteristics of reflectivity and the spatial profile of the both multilayer thickness and the core thickness. We introduce an example to design a minus reflector. Finally, the conclusions appear in section 5.

\section{Materials and Methods}

\subsection{Relationship between index variation and reflectivity}

The considered model of optical filters is illustrated in Fig. (2). The refractive index is assumed to have a small variation $\Delta n(z)$ around an averaged value $\bar{n}$ along the propagation $z$-direction of the optical wave. The wave is assumed to oscillate in the TE-polarization and is characterized by a propagation constant $\beta$.

Due to such index variation, the optical wave suffers varying reflections which sum up at the input port $(z=-L / 2)$ and constitute the filter reflection coefficient which is approximately given by the Fourier-transformation (FT)like form of $\Delta n(z)$ :

$$
r(\beta)=-j \frac{2 \pi}{\lambda_{0}} \int_{-L / 2}^{L / 2} \Delta n(z) \exp \{-2 j \beta z\} d z
$$

Therefore, for a specified profile $r(\beta)$, the spatial profile $\Delta n(z)$ of a long enough filter is given by the inverse Fourier transformation (IFT):

$$
\Delta n(z)=j \frac{\lambda}{2 \pi} \int_{-\infty}^{\infty} r(\beta) \exp \{-2 j \beta z\} d \beta
$$


The profile of index variation is assumed to have a periodic component such that the pitch is equal to $\Lambda=\lambda_{0} / 2 \bar{n}$ with $\lambda_{0}$ as the wavelength of the exciting light. Such index variation can be achieved in a multilayer filter by spatial variation of the filter material along the z-direction or to fix the material and change the layer thickness. It is then practical to change such continuous variation of $\Delta n(z)$ to a rectangular type in order to reduce the number of materials as will be discussed latter. In a waveguide filter, the index variation is achieved by corrugating the upper core interface, which then causes variation of the core thickness. The notation of index variation $\Delta n(z)$ should then change to effective index variation $\Delta n_{\text {eff }}(z)$.

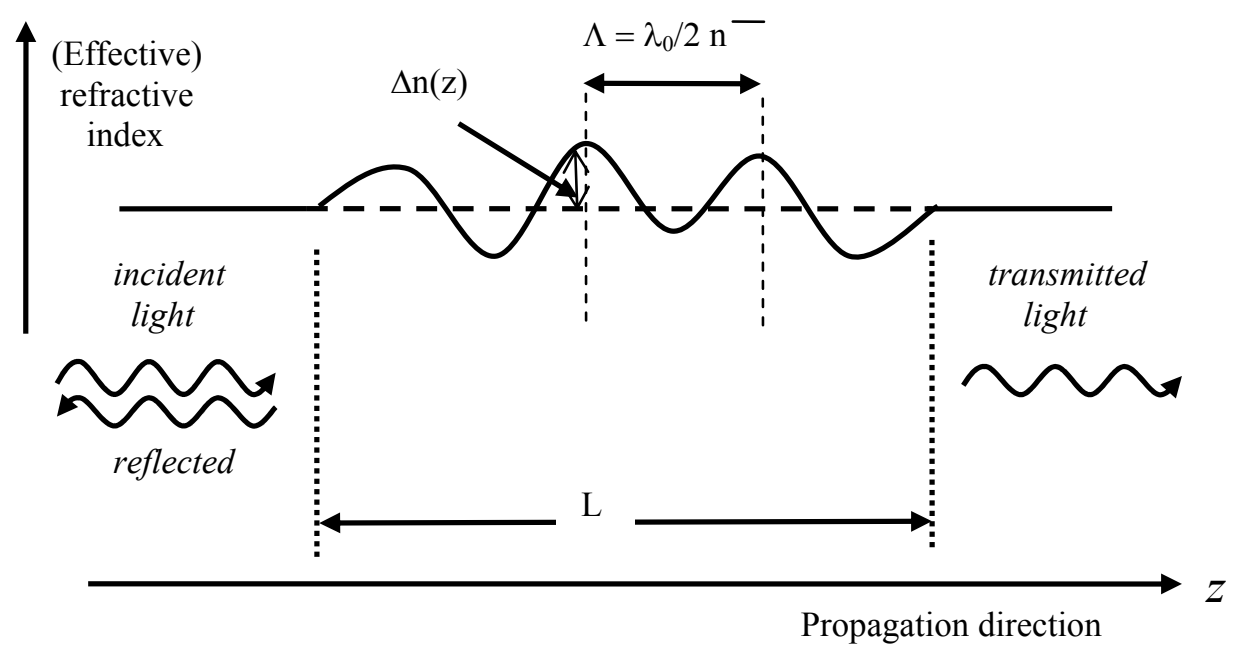

Fig. (2): Spatial profile of $\Delta n$ in an approximate analysis by the Fourier transformation.

\subsection{Multilayer Filter and analysis by the transfer-matrix method}

For the design of a high performance optical filter, it is important to determine its reflectivity and transmittance in a dielectric multi-sectioned structure. In a multilayer structure, as shown in Fig. (3), the filter consists of an alternating sequence of high and low refractive index for $N$ layers. The thickness and refractive index of the $i$-th layer are $l_{i}$ and $n_{i}$, respectively and consequently each layer is characterized by propagation impedance $Z_{(i)}=\sqrt{\mu_{0} / \varepsilon_{0}} / n_{i}$ and wavenumber $\beta_{i}=n_{i} \omega \sqrt{\varepsilon_{0} \mu_{0}}$. 

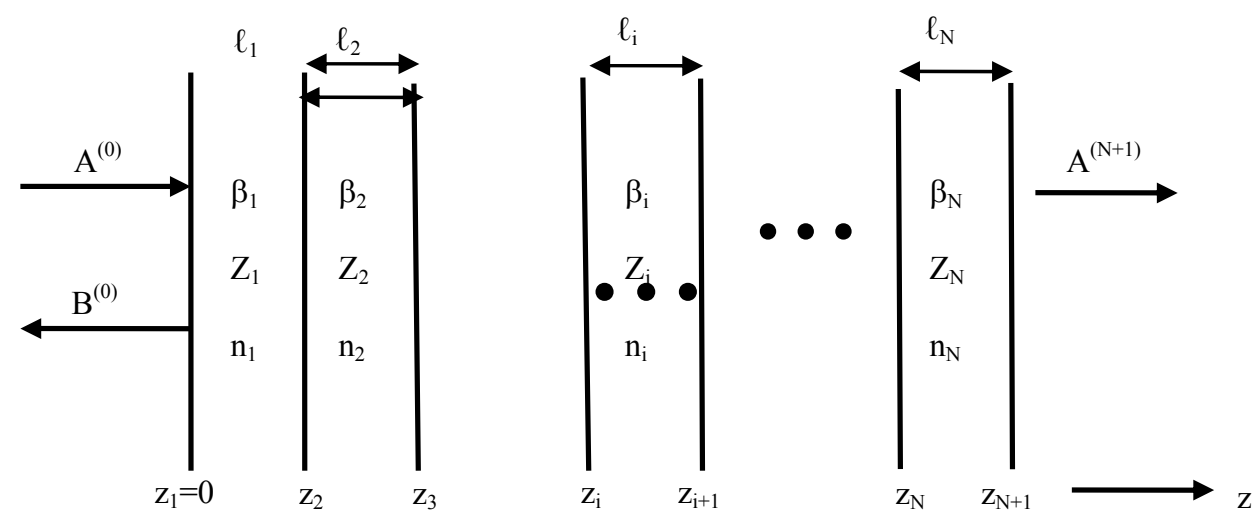

Fig. (3): Schematic design of the aperiodic multilayer optical filter.

The transfer-matrix method (TMM) is an efficient and accurate technique to analyze the propagating field in such a composite medium and calculate the reflection coefficient $[17,18]$. It simply relates the field components at the input and output ports through matrices characterizing the composing regions of the medium. Here we apply this method to calculate the reflection coefficient $r(\beta)$ that corresponds to the approximate (guide) variation of the refractive index $\Delta n(z)$ determined in the above part as the IFT of the desired spectrum.

The electric and magnetic components in the $i$-th layer are given by

$$
\begin{aligned}
& E^{(i)}(z)=A^{(i)}(z) e^{-j \beta_{i}\left(z-z_{i}\right)}+B^{(i)}(z) e^{j \beta_{i}\left(z-z_{i}\right)} \\
& H^{(i)}(z)=1 / Z_{i}\left\{A^{(i)}(z) e^{-j \beta_{i}\left(z-z_{i}\right)}-B^{(i)}(z) e^{j \beta_{i}\left(z-z_{i}\right)}\right\}
\end{aligned}
$$

where the phasor of each component is the superposition of two monochromatic plane waves counter-propagating in $z$-direction and having $e^{j \omega t}$ harmonic time dependence. According to Fig. $3, A^{(i)}(z)$ and $B^{(i)}(z)$ denote, respectively, the forward and backward field amplitudes of the wave at the interface $z=z_{i}$ in section $i$ with $z_{i-1} \leq z \leq z_{i}$.

In nonmagnetic materials, continuity of both the electric and magnetic fields is required at each interface, leading to a combination of the field components at the input side and the output side of the form

$$
\left(\begin{array}{l}
E^{(0)}\left(z_{1}\right) \\
H^{(0)}\left(z_{1}\right)
\end{array}\right)=\left(\begin{array}{ll}
S_{1} & S_{2} \\
S_{3} & S_{4}
\end{array}\right)\left(\begin{array}{l}
E^{(N+1)}\left(z_{N}\right) \\
H^{(N+1)}\left(z_{N}\right)
\end{array}\right)
$$


with

$$
\left(\begin{array}{ll}
S_{1} & S_{2} \\
S_{3} & S_{4}
\end{array}\right)=\prod_{i=1}^{N}\left(\begin{array}{cc}
\cos \left(\beta_{i} \ell_{i}\right) & j Z_{(i)} \sin \left(\beta_{i} \ell_{i}\right) \\
\left(j / Z_{i}\right) \sin \left(\beta_{i} \ell_{i}\right) & \cos \left(\beta_{i} \ell_{i}\right)
\end{array}\right)
$$

being a product matrix of the matrices characterizing the composing layers. In the external region of $z>z_{N+1}$, there is no reflection i.e. $B_{N+1}=0$ and Eq. (5) can be rewritten as

$$
\left(\begin{array}{l}
E^{(0)}\left(z_{1}\right) \\
H^{(0)}\left(z_{1}\right)
\end{array}\right)=\left(\begin{array}{ll}
S_{1} & S_{2} \\
S_{3} & S_{4}
\end{array}\right)\left(\begin{array}{c}
1 \\
\left(1 / z_{N+1}\right)
\end{array}\right) A^{N+1}
$$

At $z=z_{1}$, the reflection coefficient is defined as the ratio between the backward and the forward traveling wave:

$$
r(\beta)=\frac{B^{(0)}\left(z_{1}\right)}{A^{(0)}\left(z_{1}\right)} \equiv \frac{S_{1}+S_{2} / Z_{(N+1)}-\left(S_{3}+S_{4} / Z_{(N+1)}\right) Z_{(0)}}{S_{1}+S_{2} / Z_{(N+1)}+\left(S_{3}+S_{4} / Z_{(N+1)}\right) Z_{(0)}} .
$$

Similarly, the transmission coefficient is given by

$$
t(\beta)=\frac{A^{(N+1)}\left(z_{N+1}\right)}{A^{(0)}\left(z_{1}\right)}=\sqrt{1-|r(\beta)|^{2}} \equiv \frac{2}{S_{1}+S_{2} / Z_{(N+1)}+\left(S_{3}+S_{4} / Z_{(N+1)}\right) Z_{0}} .
$$

\subsection{Corrugated waveguide filter}

In a slab waveguide structure, as shown in Fig. 4, the effective index variation $\Delta n_{\text {eff }}(z)$ is achieved by employing a corrugation $\Delta h(z)$ on the upper interface of the core whose averaged thickness is $\bar{h}$. The considered waveguide is of an asymmetric type with refractive indices $n_{f}$ in the core, $n_{s}$ in the cladding substrate and $n_{c}$ in the cladding cover. 


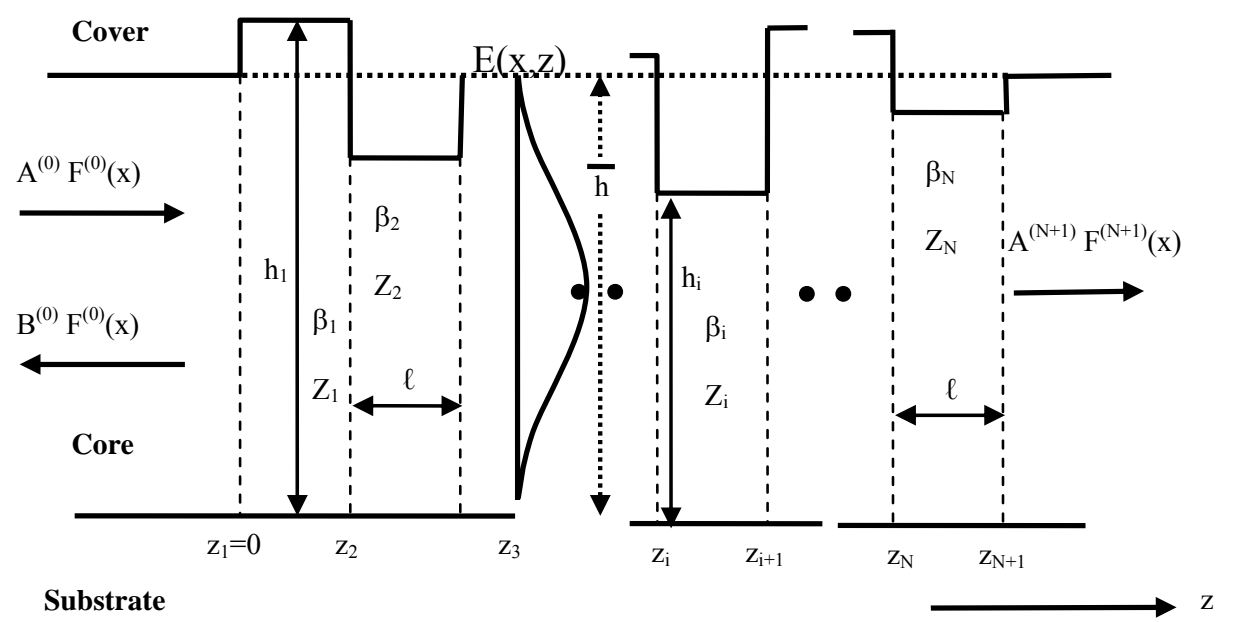

Fig. (4): Scheme of the proposed optical corrugated waveguide filter.

The light is assumed confined in the $x$-direction but diverged in the $y$ direction (no guiding structure). The waveguide is characterized by a normalized index difference $\Delta=\left(n_{f}^{2}-n_{s}^{2}\right) / 2 n_{f}^{2}$ and an asymmetry parameter $a=\left(n_{s}^{2}-n_{c}^{2}\right) /\left(n_{f}^{2}-n_{s}^{2}\right)$. The normalized frequency of the waveguide is given by:

$$
V=k_{0} h n_{f} \sqrt{2 \Delta}
$$

which determines the cutoff condition of the propagating $m$-th mode:

$$
V_{\text {cutoff }(m)}=m \pi+\tan ^{-1} \sqrt{a}
$$

where $k_{o}$ is wavenumber in free space. Therefore the thickness that supports propagation of only the fundamental mode $m=0$ (cutoff-thickness of mode $m=1$ ) satisfies the inequality

$$
h(z)<h_{\text {cutoff }(m=1)}=\frac{1}{k_{0} n_{f} \sqrt{2 \Delta}}\left\{\pi+\tan ^{-1} \sqrt{a}\right\}
$$

and is determined in terms of the normalized index difference $b(z)=\left(n_{\text {eff }}^{2}(z)-n_{s}^{2}\right) /\left(n_{f}^{2}-n_{s}^{2}\right)$ through the normalized dispersion relation [19]: 


$$
h(z)=\frac{1}{k_{0} n_{f} \sqrt{2 \Delta(1-b(z))}}\left\{\tan ^{-1} \sqrt{\frac{b(z)}{1-b(z)}}+\tan ^{-1} \sqrt{\frac{b(z)+a}{1-b(z)}}\right\}
$$

In the waveguide filter, as given in Fig. 4, the corrugated region is assumed as decomposed into $\mathrm{N}$ equal thin rectangular sections perpendicular to the propagation z-axis as shown in Fig. (4). The length and averaged thickness of the $\mathrm{i}$-th $(\mathrm{i}=1,2, \ldots, \mathrm{N})$ section are $\ell=\mathrm{Z}_{\mathrm{i}+1}-\mathrm{Z}_{\mathrm{i}}$ and $\mathrm{h}_{\mathrm{i}}$, respectively. The field components in the $\mathrm{i}$-th section are then given by:

$$
\begin{aligned}
& E^{(i)}(x, z)=\left\{A^{(i)}(z) e^{-j \beta_{i}\left(z-z_{i}\right)}+B^{(i)}(z) e^{j \beta_{i}\left(z-z_{i}\right)}\right\} F^{(i)}(x) \\
& H^{(i)}(x, z)=1 / Z_{i}\left\{A^{(i)}(z) e^{-j \beta_{i}\left(z-z_{i}\right)}-B^{(i)}(z) e^{j \beta_{i}\left(z-z_{i}\right)}\right\} E^{(i)}(x)
\end{aligned}
$$

The field distribution $F^{(i)}(x)$ is determined in terms of $h_{i}$ for the $i$-th section as [19]

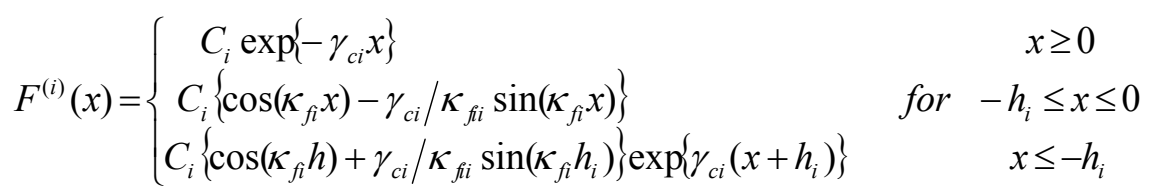

where $C_{i}$ is a normalizing constant of $F^{(i)}(x), \kappa_{f i}, \gamma_{s i}$ and $\gamma_{c i}$ are the eigenvalues characterizing the field propagation along the $x$-direction of the $i$-th element in the core, substrate and cover, respectively. They, along with $\beta_{i}=2 \pi n_{\text {eff }}^{(i)} / \lambda$ satisfy the eigenvalue relations:

$$
\begin{aligned}
& \gamma_{s i}^{2}=\beta_{i}^{2}-k_{0}^{2} n_{s}^{2} \\
& \gamma_{c i}^{2}=\beta_{i}^{2}-k_{0}^{2} n_{c}^{2} \\
& \kappa_{f i}^{2}=k_{0}^{2} n_{f}^{2}-\beta_{i}^{2} \\
& \tan \left(\kappa_{f i} h_{i}\right)=\left(\gamma_{c i}+\gamma_{s i}\right) / \kappa_{f i}\left[1-\frac{\gamma_{c i} \gamma_{s i}}{\kappa_{f i}^{2}}\right]
\end{aligned}
$$

These equations are transcendental and should be solved numerically. Application of the TMM analysis to the waveguide filter results in the same expressions (8) and (9) of the reflection and transmission coefficients of the multilayer filter. However, accuracy of such analysis gets worse if higher transverse modes are supported by the waveguide structure. 


\section{Spectral Characteristics of Optical Filter}

The optical filter can be made with a single- or a multiple-stop band, depending on the type of application. Here, we presented filters with single and double-band spectra.

\subsection{Single stop-band filter}

The desired spectrum of reflectivity $R_{0}(\beta)=\left|r_{0}(\beta)\right|^{2}$ of the single narrow-band reflector with bandwidth $\Delta \beta$ is described mathematically by the expression:

$$
r_{0}(\beta)= \begin{cases}1 & \text { for } \beta_{0}-\Delta \beta / 2 \leq \beta \leq \beta_{0}+\Delta \beta / 2 \\ 0 & \text { elsewhere }\end{cases}
$$

The spectral dependence is given in terms of the propagation constant $\beta$ rather then the wavelength $\lambda$ to make easier calculation of the index variation $\Delta \mathrm{n}(\mathrm{z})$ as the IFT of $\mathrm{r}(\beta)$ via Eq. (2). The negative wave number region $(\beta<0)$ is also considered as $r_{0}(\beta)=-r_{0}(\beta)$ to get real values for $\Delta n_{\text {eff }}(z)$. Such approximated spatial profile $\Delta \mathrm{n}_{\text {eff }}(\mathrm{z})$ is then given by :

$$
\Delta n(z)=-\frac{\Delta \beta \lambda_{0}}{\pi^{2}} \sin \left(2 \beta_{0} z\right) \operatorname{Sinc}(\Delta \beta z)
$$

which is a beating oscillating function: the fast oscillation is represented by the sine-function and the Sinc-function gives the envelope of such oscillation.

\subsection{Double-band (minus) reflection filter}

A minus-reflection filter can be described by the two-rejection band expression of the reflection coefficient $\mathrm{r}(\beta)$ :

$$
r_{0}(\beta)= \begin{cases}1 & \text { for } \beta_{0}-(\Delta \beta+\zeta / 2) \leq \beta \leq \beta_{0}-\zeta / 2, \beta_{0}+\zeta / 2 \leq \beta \leq \beta_{0}+(\Delta \beta+\zeta / 2) \\ 0 & \text { elsewhere }\end{cases}
$$

This type of optical filter is very important for WDM applications. The width of the two stop bands $\Delta \beta$ can be made much wider than the separation in between in such a way that it can be used for multiple-channel WDM. The profile of $\Delta \mathrm{n}(\mathrm{z})$ is then given by the form

$$
\Delta n(z)=-\frac{4 \Delta \beta \lambda_{0}}{\pi \omega \sqrt{\mu \varepsilon_{0}}} \sin \left(2 \beta_{0} z\right) \operatorname{Sinc}(\Delta \beta z) \cos [(\Delta \beta+\zeta) z]
$$


The continuous variation of $\Delta \mathrm{n}(\mathrm{z})$ described by Eqs. (20) and (22) can be transformed to a rectangular type by means of a Fourier expansion:

$$
\sin \left(2 \beta_{0} z\right) \approx\left\{\begin{array}{cl}
\pi / 4 & \text { for } \mathrm{z}_{2 \mathrm{~m}} \leq z \leq z_{2 m+1} \\
-\pi / 4 & \text { for } \mathrm{z}_{2 \mathrm{~m}+1} \leq z \leq z_{2 m+2}
\end{array}\right.
$$

Since the forms (20) and (22) of $\Delta \mathrm{n}(\mathrm{z})$ are obtained for long-enough filters, a large number of materials will be required to realize such a multilayer filter if the layer thickness is kept constant $\ell_{i}=\ell$. For more practical device, the filter can be designed with pairs of two materials with refractive indices $n_{H}$ and $\mathrm{n}_{\mathrm{L}}$ and $\ell_{H}^{(i)}$ and $\ell_{L}^{(i)}$ satisfying the transfer-matrix condition [15]

$$
n_{H} \ell_{H}+2 n_{L} \ell_{L}=\lambda_{0} / 2
$$

Such filter design requires transformation of the negative part of $\Delta n(z)$ to a positive one by:

$$
\Delta n^{\prime}(z)=\left\{\begin{array}{cc}
2 \Delta \mathrm{n} & \text { for } \mathrm{n}(\mathrm{z}) \geq \bar{n} \\
0 & \text { for } \mathrm{n}(\mathrm{z}) \prec \bar{n}
\end{array}\right.
$$

The thickness of the higher-index layer $\ell_{H}^{(i)}$ is determined by the equation [15]

$$
L_{H}^{(i)}=\frac{\lambda_{0}}{2 \pi n_{H}}\left[\frac{\pi}{2}-\cos ^{-1}\left(\frac{\Delta n^{\prime}(z)}{n_{H}-n_{L}}\right)\right]
$$

\section{Results and discussion}

\subsection{Limitation of periodic Bragg reflectors}

The solid curve in Fig. (5) shows the calculated reflectivity spectrum $R(\lambda)$ for the case of periodic distributed-Bragg-reflector (DBR) using Eq. (8). The reflector consists of $25 \mathrm{GaAs}$ and AlAs mirror pairs of refractive indices 3.0 and 3.6, respectively. The curve has a broad spectral plateau (stop band) around the central Bragg wavelength where $R(\lambda) \sim 100 \%$. The width of the stop band is nearly $100 \mathrm{~nm}$ which represents a characteristic feature of the DBR as a resonator for laser production, namely the vertical cavity surface emitting lasers [20]. One drawback of the DBR method is the existence of side lobs around the stop band of reflectivity $\geq 80 \%$. The corresponding aperiodic design is ahieved by employing Eq.(20) of the approximated profile of $\Delta n(z)$ after modulation by a parameter $u=18.2$ and calculating the reflection coefficient via Eq. (8). 
The obtained reflectivity spectrum is plotted in Fig. 5 with the dashed curve. This curve seems to be identical with that obtained from the periodic DBR especially in the stop band region, but the side lobes are dramatically suppressed. Having done the reflectivity spectrum, the modulated index profile is shown in Fig. 6. The profile reaches its maximum and minimum value of 3.6 and -3.6 , respectively at the adjacent central layers and then oscillates around the average refractive index $n=3.3$ with a slightly degradation from the center to the outer edges at which the refractive index is $0.2 \%$ lower. Another typical drawback of the periodic filter design is the difficulty to obtain a narrow stop band around $1 \mathrm{~nm}$ which will be overcome by the aperiodic design as given in the following subsections.

\subsection{Dielectric aperiodic multilayered reflector}

In order to decrease the bandwidth of the filter to the desired value, aperiodic design should be achieved. In this case, the bandwidth of the assumed spectrum in Eq. (19) is set as $\beta=66.10^{-5} \beta_{0}$ which corresponds to $\Delta \lambda \sim 1 \mathrm{~nm}$. This case of narrow-band filter requires a large number of layers (i.e., long-enough filter) because the accuracy of calculating the reflectivity via the approximate FT-analysis deteriorates with deceasing the width $\Delta \beta$. The approximated power reflectivity spectra for two lengths of 1 and 2mm are shown in Fig.7(a).

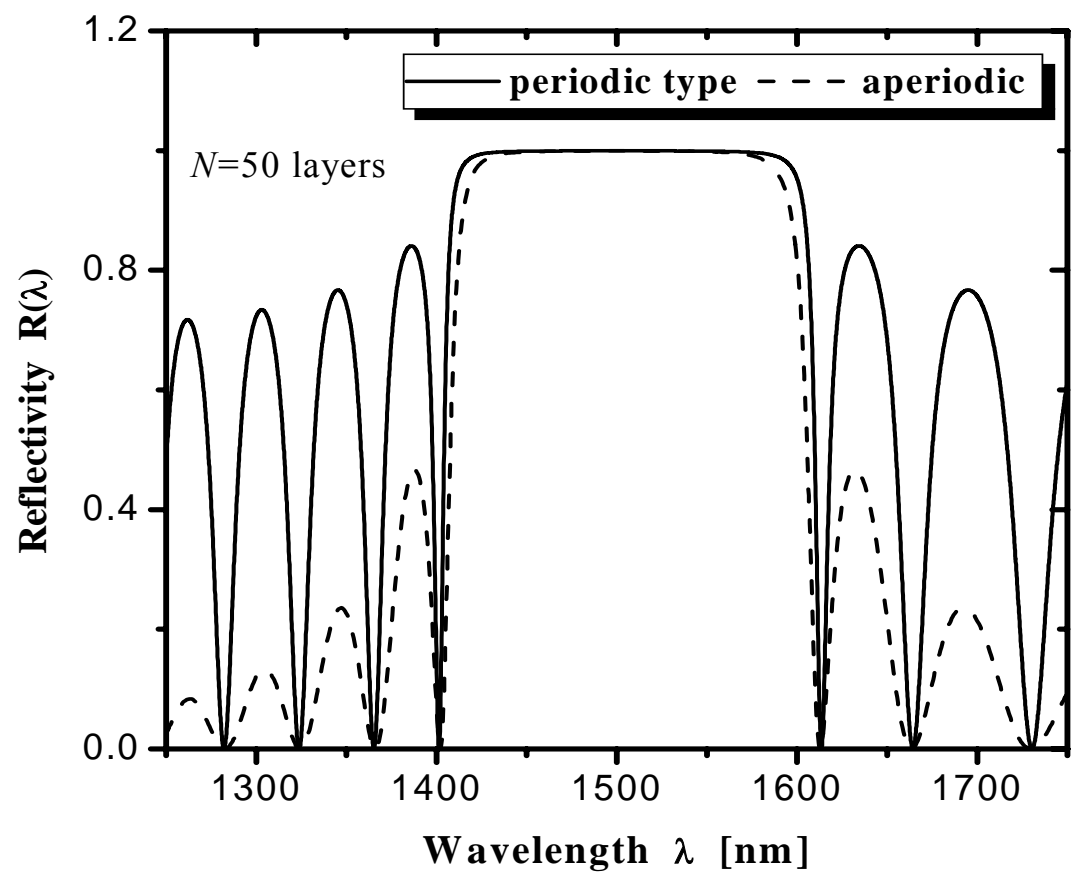

Fig. (5): Comparison between the reflectivity spectra of periodic DBR and an aperiodic filter. 


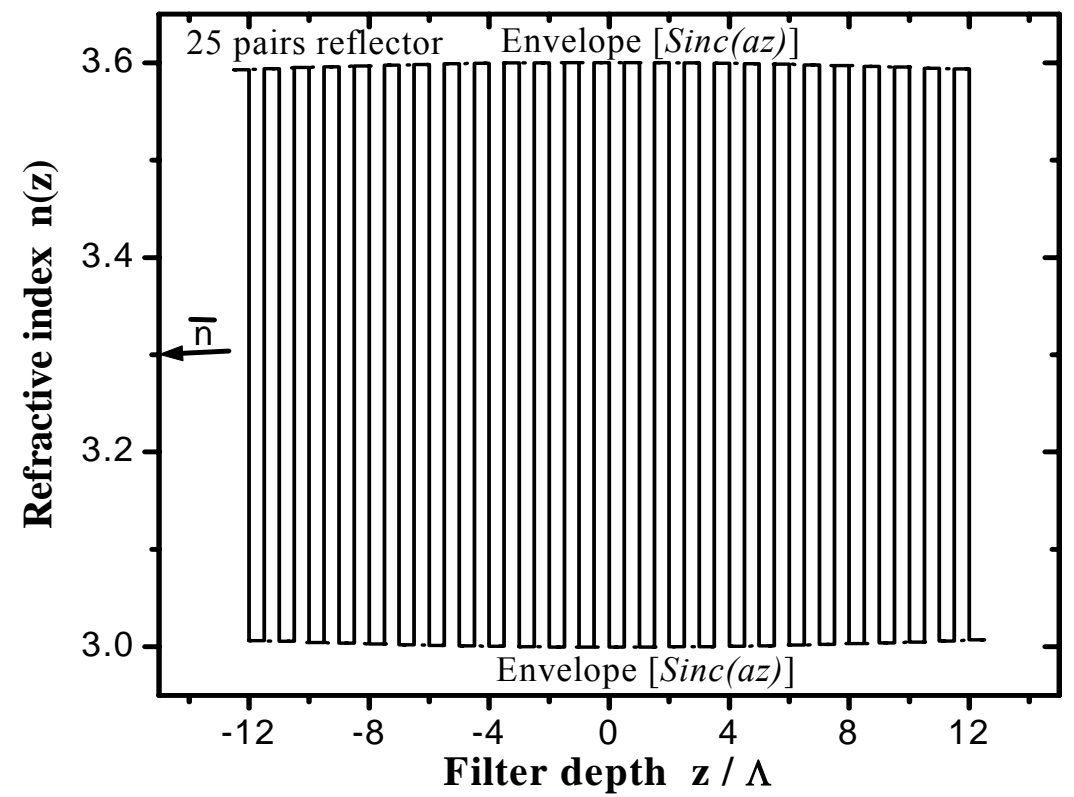

Fig. (6): Modulated refractive index profile with $u=18.2$.

Of course, one sees the effect of increasing the filter length on the enhancing maximum reflectivity. This is attributed to the increase of the number of layers and consequently the increase of the reflected wave amplitudes. The approximated reflectivity spectra at 1 and $2 \mathrm{~mm}$ filter lengths have $44 \%$ and $68 \%$ maximum values, respectively, with width larger than the set value of 1 $\mathrm{nm}$. This means that there still requirements to improve these spectra to fit the assumed one especially in the stop band region.
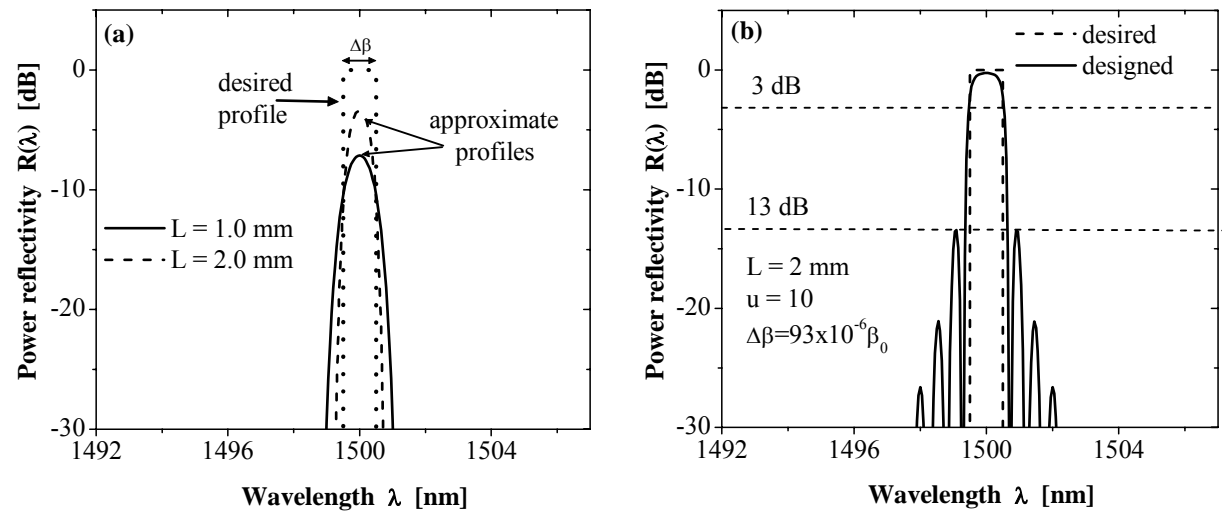

Fig. (7): Comparison between (a) the power reflectivity of aperiodic optical filter with $1 \mathrm{~nm}$ stop band for different filter lengths, and (b) the fitreflectivity spectrum and the assumed one. 
This is realized for the case of $2 \mathrm{~mm}$ filter length by reducing the band width to $\Delta \beta=93 \cdot 10^{-6} \beta_{0}$ and multiplying Eq. (20) by a modulation parameter $u=10$. The resulted reflectivity spectrum is illustrated in Fig. $7(\mathrm{~b})$ by the solid curve which fits the desired one in the stop band region (dotted line) with maximum reflectivity of $97 \%,-3 \mathrm{~dB}$ bandwidth $\sim 1 \mathrm{~nm}$ and side lobes lower than $-13 \mathrm{~dB}$. The difference between the approximated index profile $\Delta n(z)$ and the modified one is clearly shown in Fig. 8(a), where the envelope of the former shows a higher decrease from the center to the outer edges of the filter than the latter.
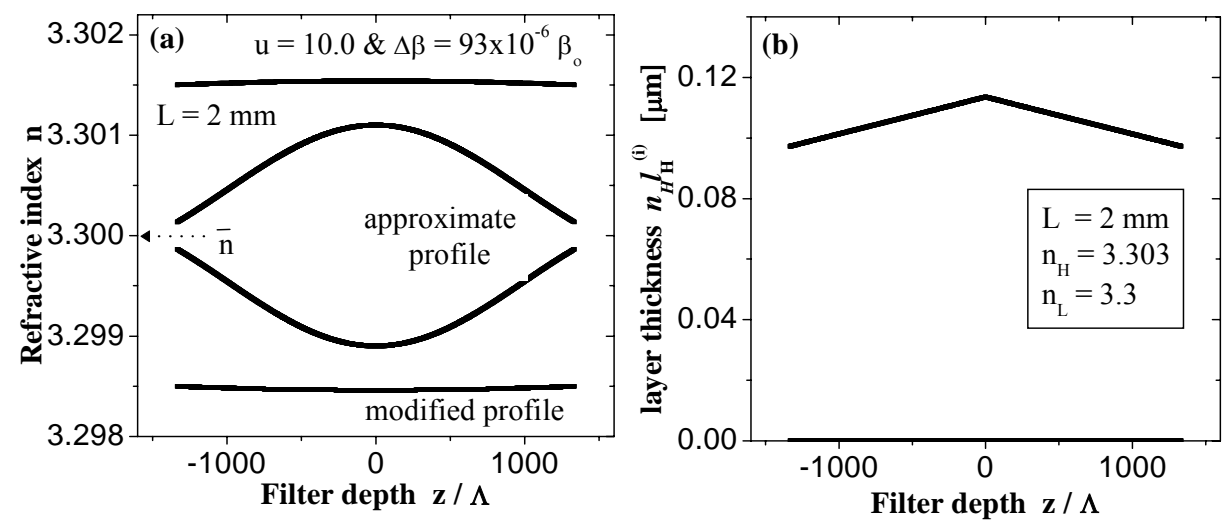

Fig. (8): Spatial profiles of (a) the approximate and modulated profiles of index profile $\Delta n(z)$ for optical lengths of 1 and $2 \mathrm{~mm}$, and (b) one layer thickness $l_{H}^{(i)}$ for the $2.0 \mathrm{~mm}$ optical length.

In the case of $2 \mathrm{~mm}$ length filter, the number of layers with different refractive indices is 5334 . That is, one needs a huge number of materials to design such a filter. Therefore, we change the design to utilize pairs of two materials $\left(n_{H}\right.$ and $\left.n_{L}\right)$ with thicknesses $\ell_{H}^{(i)}$ and $\ell_{L}^{(i)}$ as described in subsection 2.2. This design is easier and commercial from the practical point of view. Figure $8(\mathrm{~b})$ illustrate the spatial variation of one thickness, namely $\ell_{H}^{(i)}$.

\subsection{Corrugated waveguide reflector}

We use the material system $\mathrm{Al}_{\mathrm{x}} \mathrm{Ga}_{1-\mathrm{x}} \mathrm{As} / \mathrm{GaAs}$ in the considered waveguide structure. The core is made of GaAs which has a bandgap energy of $1.424 \mathrm{eV}$ and wavelength $\lambda_{\mathrm{g}} \sim 0.78 \mu \mathrm{m}$ which is much shorter than the exciting wavelength $\lambda_{0}=1.5 \mu \mathrm{m}$. That is the material is treated as a dielectric for the incident light. The composition $\mathrm{x}$ of the Arsenide is chosen as 0.1 in the substrate and 0.2 in the cover. Since the refractive index in this binary semiconductor changes with $\mathrm{x}$ as [21]: 


$$
n=3.59-0.71 x+0.091 x^{2}
$$

the chosen composition values result in refractive indices of $\mathrm{n}_{\mathrm{f}}=3.59>\mathrm{n}_{\mathrm{s}}=3.524>\mathrm{n}_{\mathrm{c}}=3.43$ which satisfy the universal condition of waveguiding [19]. The relative refractive index is $\Delta=2 \%$ and the asymmetry parameter is $\mathrm{a}=0.955$. The film thickness of the smooth-interface waveguide is set as $\bar{h}=1.0 \mu \mathrm{m}$, which corresponds to the normalized frequency $\tan ^{-1} \sqrt{a}=0.774<V=2.986<V_{\text {cutoff }(m=1)}=3.916$ i.e., $\quad$ the considered waveguide supports propagation of only the fundamental $\mathrm{TE}_{0}$ mode. This result is confirmed by solving Eqs. (17) and (18) numerically, which results in a single solution characterizing the $\mathrm{TE}_{0}$ mode. The obtained eigenvalues are $\kappa_{\mathrm{f}}=1.398 \times 10^{6} \mathrm{~m}^{-1}, \gamma_{\mathrm{c}}=3.652 \times 10^{6} \mathrm{~m}^{-1}, \gamma_{\mathrm{s}}=2.233 \times 10^{6} \mathrm{~m}^{-1}$ and $\beta=1.491 \times 10^{7} \mathrm{~m}^{-1}$ which correspond to $\bar{n}_{\text {eff }}=3.56$ and $\bar{b}=0.57 \mathrm{~b}=0.57$. The corresponding pitch of the corrugation is then $\Lambda=0.211 \mu \mathrm{m}$.

The corrugation structure of the core thickness $h(z)$ corresponding to the fit-reflectivity spectrum $R(\beta)$ shown in Fig. 7(b) is plotted in Fig. (9). This figure plots only the envelope of the spatial variation noting that each point represents amplitude of the corresponding rectangular section of length $\ell \approx \Lambda / 2$. It is worth to note that the maximum value of the core thickness is $\mathrm{h}_{\max }=1.031 \mu \mathrm{m}$ which is less than the cutoff thickness $\mathrm{h}_{\text {cutoff(m=1) }}=1.311 \mu \mathrm{m}$ of mode $\mathrm{m}=1$ as determined by Eq. (12). That is, the transverse modes are restricted to the fundamental one through the corrugated region.

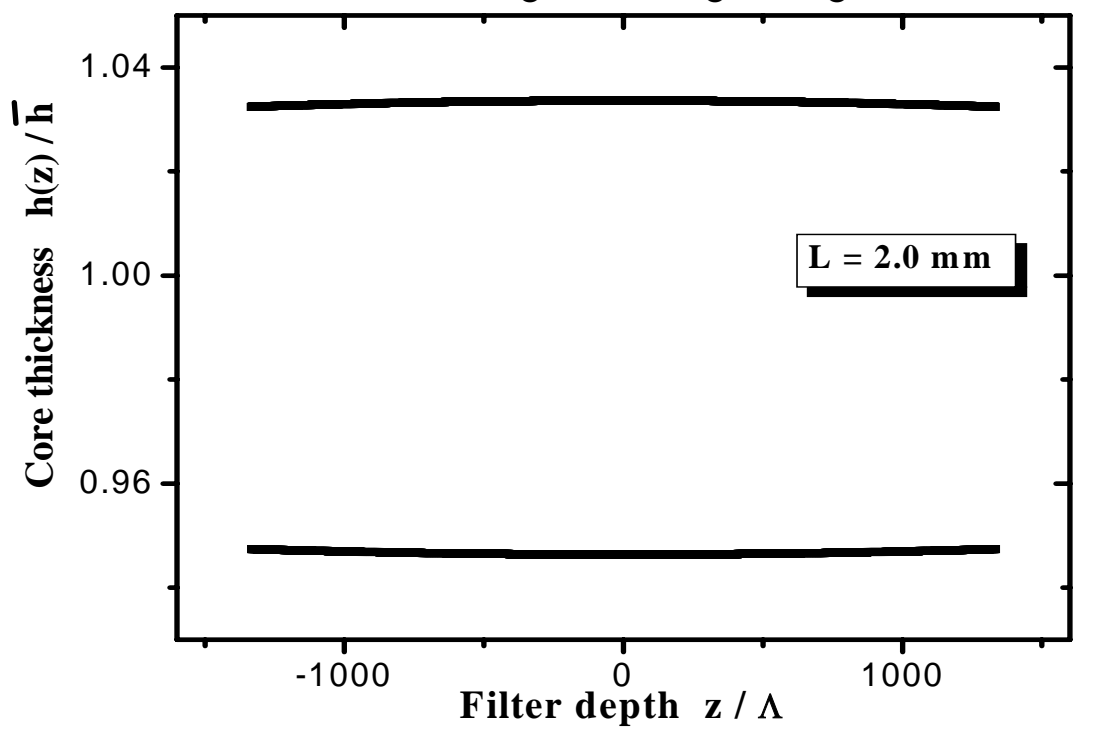

Fig. (9): Spatial profile of the core thickness. 
It might be useful to note that the length of the corrugated part (or the number of corrugated sections) is not a critical problem for fabrication of this type of structure like the case of multilayer reflector. The corrugation can be formed on the top core-interface by means of the electron beam-lithography technique [21]. The corrugation pitch can be increased to alleviate fabrication of the corrugation structure by choosing low-index materials for the waveguide (for silica, $\Lambda=0.51 \mu \mathrm{m}$ ).

\subsection{Characteristics of a double-band (minus) reflector}

This example shows the superiority of aperiodic structures over periodic ones to achieve more than one stop band. In this example of doubleband reflectivity described by Eq. (21), the bandwidth is set as small as $\Delta \beta=23.10^{-4} \beta_{0}$ and the band separation is set as $\zeta=\Delta \beta / 2$, which correspond to wavelengths of 3.5 and $1.75 \mathrm{~nm}$, respectively. The approximated profile of $\Delta n(z)$ in Eq. (22) is modulated by a factor of $u=3$ in order to fit the calculated reflectivity $R(\lambda)$ with the desired one $R_{0}(\lambda)$. Figure 10 indicates good fit of the spectra is shown in Fig. 10 in the higher values of $R(\lambda)$, and Fig. 11(a) plots the designed spatial profile of the layer thickness $\ell_{H}^{(i)}$. The bandwidth of the calculated $R(\lambda)$ gets wider at lower reflectivity values at the expense of the band separation $\zeta$. Surprisingly, such effect reduces the minus-reflectivity (transmission) around the central wavelength $\lambda_{0}=1.5 \mu \mathrm{m}$ to $\sim 0.6 \mathrm{~nm}$ at $T(\lambda)=-$ $3 \mathrm{~dB}$, as shown in the zoomed figure in Fig. 10. Such spectrum is a favorable property for use as transmission filter in WDM. It is worth to note that the fit of reflectivity spectra is improved by increasing $\zeta$ at the expense of $\Delta \beta$.

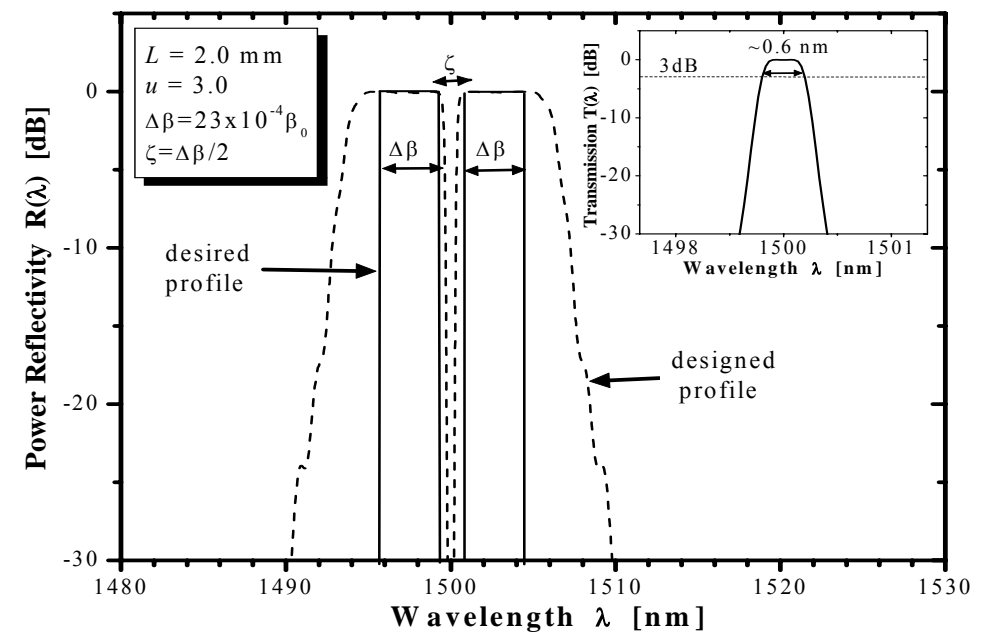

Fig. (10): Comparison between the calculated and desired power reflection of aperiodic optical filter. Good fit is seen of higher values of $R(\lambda)$ and narrow band transmission is obtained. The inset represents the transmission spectrum. 
Such transmission filter can be realized by a corrugated waveguide structure. The profile of the core thickness $h(z)$ is shown in Fig. 11(b), which is much deeper than the single-band case. In this case, higher transverse modes are expected to propagate down the filter. Such profile amplitude can be reduced by increasing the asymmetry parameter $a$.
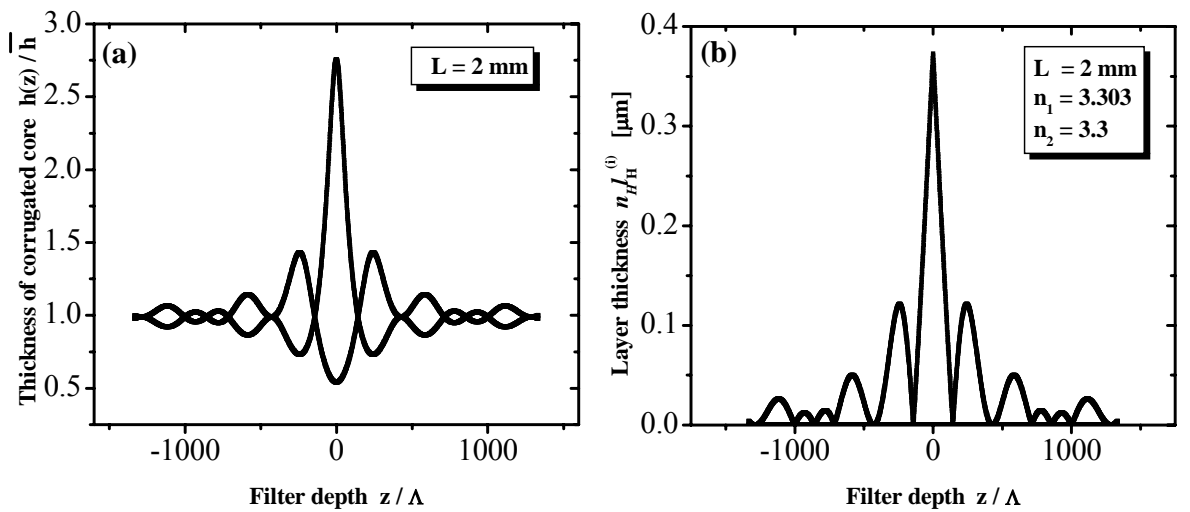

Fig. (11): Spatial profiles of: (a) the core thickness $h(z)$ of the waveguide structure and (b) the layer thickness $\ell_{\mathrm{H}}{ }^{(\mathrm{i})}$ of the multilayer structure for the double-band filter with optical length of $2.0 \mathrm{~mm}$.

\section{Conclusions:}

We proposed reflection filters with bandwidth $\leq 1 \mathrm{~nm}$ for use in wavelength-division-multiplexed optical communications. The filter was demonstrated in both multilayer and waveguide structures. The present synthesis model employs aperiodic variation of the refractive index. We achieved reflectors with $97 \%$ reflectivity, bandwidth of $\leq 1 \mathrm{~nm}$ and low sidelobes $(<-13 \mathrm{~dB})$ using pairs of GaAs-AlAs multilayers and GaAs/AlGaAs waveguides with a corrugated structure on the upper interface of the core. We also demonstrated accomplishment of two-band reflectors for use as minus (transmission) filters. The $3 \mathrm{~dB}$ band width of this filter is $0.5 \mathrm{~nm}$ with $99 \%$ maximum transmission.

\section{References:}

1. M. M. Liu, "Principles and applications of optical communications", (McGraw-Hill, New York, 1966)

2. Yariv: IEEE J. Quantum Electron. QE-9, 919, (1973).

3. F. W. Dabby, M. A. Saifi and A. Kestenbaum: Appl. Phys. Lett. 22, 190, (1973).

4. R. Schubert: J. Appl. Phys. 45, 209, (1974). 
5. D. C. Flanders, H. Kogelnik, R. V. Schmidt and C. V. Shank: Appl. Phys. Lett. 24, 194, (1974).

6. R. V. Schmidt, D. C. Flanders, C. V. Shank and R. D. Standley: Appl. Phys. Lett. 25, 651 (1974).

7. S. Kim and C. G. Fonstad, IEEE J. Quantum Electron. QE-15, 1405, (1979).

8. A. Bakhtazad, H. Abiri and M. H. Rahnavard: J. Lightwave Tech. 13, 1780, (1995).

9. Hill K. O., Appl. Opt. 13, 1853, (1974).

10. S. Hong, J. B. Shellan, A. C. Livanos, A. Yariv and A. Katzir, Appl. Phys. Lett. 4, 276, (1977).

11. J. B. Shellan, C. S. Hong and A. Yariv: Optics Commun. 23 (1977) 398.

12. Y. Shibata, T. Tamamura, S. Oku and Y. Kondo: IEEE Photonics Tech. Lett. 6, 1222, (1994).

13. K. A. Winick and J. R. Roman, IEEE J. Quantum Electron. 26, 1918, (1990).

14. M. Ahmed, M. Yamada and Y. Yamane, Opt. Rev. 3, 345, (1996).

15. M. Yamada and Y. Yamane, Opt. Rev. 3, 512, (1996).

16. M. Ahmed and M. Yamada, Opt. Rev. 4, 402, (1997).

17. M. Born and E. Wolf, "Principles of Optics", 6-th ed., Pergamon Press, Oxford (1989).

18. P.Yeh, "Optical Waves in Layered Media", J. Wiley and Sons, New York, (1988).

19. R. Pollock, "Fundamentals of Optoelectronics", McGraw-Hill, New York, (1995).

20. K. J. Ebeling, Integrated Optoelectronics, (Springer-Verlag, Berlin, 1993).

21. Y. Suematsu and A. R. Adams, "Handbook of semiconductor lasers and photonic integrated circuits", Chapman and Hall, London (1994) 